Article

\title{
On Hyperstability of the Cauchy Functional Equation in $n$-Banach Spaces
}

\author{
Janusz Brzdęk ${ }^{1, *(1)}$ and El-sayed El-hady 2,3 (i) \\ 1 Faculty of Applied Mathematics, AGH University of Science and Technology, Mickiewicza 30, \\ 30-059 Kraków, Poland \\ 2 Mathematics Department, College of Science, Jouf University, Sakaka P.O. Box 2014, Saudi Arabia; \\ eaelhady@ju.edu.sa \\ 3 Basic Science Department, Faculty of Computers and Informatics, Suez Canal University, \\ Ismailia 41522, Egypt \\ * Correspondence: brzdek@agh.edu.pl
}

Received: 18 September 2020; Accepted: 26 October 2020; Published: 30 October 2020

Abstract: We present some hyperstability results for the well-known additive Cauchy functional equation $f(x+y)=f(x)+f(y)$ in $n$-normed spaces, which correspond to several analogous outcomes proved for some other spaces. The main tool is a recent fixed-point theorem.

Keywords: hyperstability; cauchy functional equation; $n$-norm; $n$-Banach space

\section{Introduction}

The issue of stability of functional equations has been motivated by a problem raised by S.M. Ulam (see [1]), which at present can be understood in the following way:

When is it true that a function satisfying a certain property (e.g., equation) approximately must be close to a function satisfying the property exactly?

For more details and historical background, we refer to [2], which is the first monograph on the subject (see also [3]). An interesting discussion on various possible approaches to such stability has been presented in [4] (see also [5]); we use the one described in our Definitions 1 and 2.

Problems of that type are very natural for difference, differential, functional and integral equations and many examples of recent results concerning their stability as well as further references can be found in [6,7].

Roughly speaking, we say that a given functional equation is stable in some class of functions if any function from that class, satisfying the equation approximately, is near an exact solution of the equation. One of the classical outcomes in this area is the following theorem (see Theorems 3.1 and 3.4 of [8]) concerning stability of the well-known Cauchy functional equation on a restricted domain $(\mathbb{N}$ denotes the set of positive integers).

Theorem 1. Let $E_{1}$ and $E_{2}$ be normed spaces, $c \geq 0$ and $p \neq 1$ be real numbers and $\varnothing \neq X \subset E_{1} \backslash\{0\}$. Let $f: X \rightarrow E_{2}$ be a mapping such that

$$
\left\|f\left(x_{1}+x_{2}\right)-f\left(x_{1}\right)-f\left(x_{2}\right)\right\| \leq c\left(\left\|x_{1}\right\|^{p}+\left\|x_{2}\right\|^{p}\right), \quad \text { for every } x_{1}, x_{2} \in X \text { with } x_{1}+x_{2} \in X .
$$

Then the following two statements are valid. 
(i) If $p<0, X=-X$ and there exists $k_{0} \in \mathbb{N}$ such that $k x \in X$ for every $x \in X$ and every $k \in \mathbb{N}$ with $k \geq k_{0}$, then $f$ is additive on $X$ :

$$
f\left(x_{1}+x_{2}\right)=f\left(x_{1}\right)+f\left(x_{2}\right), \quad \text { for every } x_{1}, x_{2} \in X \text { with } x_{1}+x_{2} \in X .
$$

(ii) If $p \geq 0, X=2 X$ and $E_{2}$ is complete, then there exists a unique mappping $g: X \rightarrow E_{2}$ that is additive on $X$ and

$$
\|f(x)-g(x)\| \leq \frac{c}{\left|1-2^{p-1}\right|}\|x\|^{p}, \quad \text { for every } x \in X
$$

Let us mention that the assumption $p \neq 1$ is necessary (see [9]) and estimate (3) is the best possible in the general case (see [3] for more details and [10] for a related result). If $p=0$ and $X=E_{1} \backslash\{0\}$, then we obtain from Theorem 1 the result of Hyers [1].

Similar outcomes, but with (1) replaced by the inequality

$$
\left\|f\left(x_{1}+x_{2}\right)-f\left(x_{1}\right)-f\left(x_{2}\right)\right\| \leq c\left\|x_{1}\right\|^{q}\left\|x_{2}\right\|^{p}, \quad \text { for every } x_{1}, x_{2} \in X \text { with } x_{1}+x_{2} \in X,
$$

with some real numbers $p$ and $q$, have been obtained in [11-13] (see Section 3 for more details).

Clearly, the concept of an approximate solution and the idea of nearness of two functions can be understood in many nonstandard ways, depending on the needs and tools available in a particular situation. One of such non-classical measures of distance can be created using the notion of $n$-norm, introduced by A. Misiak [14]. We refer to [15] for several examples of investigations of stability of functional equations in the $n$-normed spaces.

In this paper, we present two possible extensions of the results in [13] to the case of n-normed spaces.

\section{Preliminaries}

The notion of $n$-normed space is an extension of those of the classical normed space and of the 2-normed space defined by Gähler [16] (cf., e.g., [17-20]).

Let us now recall some basic definitions and facts concerning $n$-normed spaces (for more details we refer the reader to [15]; see also [14,21-23]).

Let $n \in \mathbb{N}, X$ be a real linear space, which is at least $n$-dimensional, and $\|\cdot, \ldots, \cdot\|$ be a function mapping $X^{n}$ into $\mathbb{R}_{+}$(the set of non-negative reals) that satisfies the following conditions:

(C1) $\left\|x_{1}, \ldots, x_{n}\right\|=0$ if and only if $x_{1}, \ldots, x_{n}$ are linearly dependent,

(C2) $\left\|x_{1}, \ldots, x_{n}\right\|$ is invariant under permutation of $x_{1}, \ldots, x_{n}$,

(C3) $\left\|\beta x_{1}, \ldots, x_{n}\right\|=|\beta|\left\|x_{1}, \ldots, x_{n}\right\|$,

(C4) $\left\|x+y, x_{2}, \ldots, x_{n}\right\| \leq\left\|x, x_{2}, \ldots, x_{n}\right\|+\left\|y, x_{2}, \ldots, x_{n}\right\|$

for every $\beta \in \mathbb{R}$ (the set of reals) and $x, y, x_{1}, \ldots, x_{n} \in X$. Then $\|\cdot, \ldots, \cdot\|$ is called an $n$-norm on $X$ and the pair $(X,\|\cdot, \ldots, \cdot\|)$ is said to be an $n$-normed space.

If $n>1$ and $(X,\langle\cdot, \cdot\rangle)$ is a real inner product space, which is at least $n$-dimensional, then the formula

$$
\left\|x_{1}, \ldots, x_{n}\right\|_{S}:=\operatorname{abs}\left(\left|\begin{array}{cccc}
\left\langle x_{1}, x_{1}\right\rangle & \left\langle x_{1}, x_{2}\right\rangle & \ldots & \left\langle x_{1}, x_{n}\right\rangle \\
\vdots & \vdots & \ddots & \vdots \\
\left\langle x_{n}, x_{1}\right\rangle & \left\langle x_{n}, x_{2}\right\rangle & \ldots & \left\langle x_{n}, x_{n}\right\rangle
\end{array}\right|\right)^{1 / 2}, \quad \text { for every } x_{1}, \ldots, x_{n} \in X
$$

defines an $n$-norm on $X$, where $\operatorname{abs}(x)$ means the absolute value of a real number $x$.

If $X=\mathbb{R}^{n}$ with the usual inner product, then in this way we obtain the Euclidean $n$-norm on $\mathbb{R}^{n}$, which also can be expressed by

$$
\left\|x_{1}, \ldots, x_{n}\right\|_{E}=\left|\operatorname{det}\left(x_{i j}\right)\right|^{1 / 2}, \quad \text { for every } x_{i}=\left(x_{i 1}, \ldots, x_{i n}\right) \in \mathbb{R}^{n} \text { and } i \in 1, \ldots, n \text {, }
$$


where

$$
\operatorname{det}\left(x_{i j}\right)=\left|\begin{array}{cccc}
x_{11} & x_{12} & \ldots & x_{1 n} \\
\vdots & \vdots & \ddots & \vdots \\
x_{n 1} & x_{n 2} & \ldots & x_{n n}
\end{array}\right| .
$$

If $(X,\|\cdot, \ldots, \cdot\|)$ is an $n$-normed space and $y_{2}, \ldots, y_{n} \in X$, then (C4) implies that

$$
\left\|\sum_{i=1}^{k} z_{i}, y_{2}, \ldots, y_{n}\right\| \leq \sum_{i=1}^{k}\left\|z_{i}, y_{2}, \ldots, y_{n}\right\|, \quad \text { for every } k \in \mathbb{N} \text { and } z_{1}, \ldots, z_{k} \in X,
$$

and the function $X^{2} \ni\left(x_{1}, x_{2}\right) \rightarrow\left\|x_{1}-x_{2}, y_{2}, \ldots, y_{n}\right\|$ is non-negative.

Let us recall that a sequence $\left(x_{k}\right)_{k \in \mathbb{N}}$ of elements of an $n$-normed space $(X,\|\cdot, \ldots, \cdot\|)$ is called a Cauchy sequence if

$$
\lim _{k, l \rightarrow \infty}\left\|x_{k}-x_{l}, y_{2}, \ldots, y_{n}\right\|=0, \quad \text { for every } y_{2}, \ldots, y_{n} \in X,
$$

whereas $\left(x_{k}\right)_{k \in \mathbb{N}}$ is said to be convergent if there exists an element $x \in X$ (called the limit of this sequence and denoted by $\lim _{k \rightarrow \infty} x_{k}$ ) with

$$
\lim _{k \rightarrow \infty}\left\|x_{k}-x, y_{2}, \ldots, y_{n}\right\|=0, \quad \text { for every } y_{2}, \ldots, y_{n} \in X
$$

An $n$-normed space in which every Cauchy sequence is convergent is called $n$-Banach space. Moreover, we have the following property stated in [23] (see also [15]).

Lemma 1. Let $(X,\|\cdot, \ldots, \cdot\|)$ be an n-normed space. If $\left(x_{k}\right)_{k \in \mathbb{N}}$ is a convergent sequence of elements of $X$, then

$$
\lim _{k \rightarrow \infty}\left\|x_{k}, y_{2}, \ldots, y_{n}\right\|=\left\|\lim _{k \rightarrow \infty} x_{k}, y_{2}, \ldots, y_{n}\right\|, \quad \text { for every } y_{2}, \ldots, y_{n} \in X .
$$

Remark 1. It follows from (C1) that if $(X,\|\cdot, \ldots, \cdot\|)$ is an $n$-normed space, $z_{1}, \ldots, z_{n} \in X$ are linearly independent, $x \in X$ and

$$
\left\|x, w_{2}, \ldots, w_{n}\right\|=0, \quad \text { for every } w_{2}, \ldots, w_{n} \in\left\{z_{1}, \ldots, z_{n}\right\},
$$

then $x=0$.

Finally, let us also mention that H. Gunawan and M. Mashadi [21] showed that from every $n$-norm one can derive an $(n-1)$-norm and thus, finally, a standard norm (cf. our Remark 2). More information on the $n$-normed spaces and some problems investigated in them (among others in fixed-point theory) can be found for instance in $[15,22-28]$.

\section{Hyperstability Results}

In the rest of the paper we assume that $m \in \mathbb{N}$ and $(Y,\|\cdot, \ldots, \cdot\|)$ is an $(m+1)$-normed space. For simplicity of the notation we write

$$
\|z, y\|:=\left\|z, y_{1}, \ldots, y_{m}\right\|, \quad \text { for every } z \in Y \text { and } y=\left(y_{1}, \ldots, y_{m}\right) \in Y^{m} .
$$

Moreover, if $A$ and $B$ are nonempty sets, then $B^{A}$ denotes the family of all mappings from $A$ into $B$.

The name of Ulam has been associated with various definitions of stability (see $[2,4,23,29]$ ). The following one, formulated for the $n$-normed spaces, describes our considerations in this paper. 
Definition 1. Let $(P,+)$ be a groupoid $\left(P \neq \varnothing\right.$ is a set endowed with a binary operation $\left.+: P^{2} \rightarrow P\right), T \neq \varnothing$ be a subset of $P, E \subset \mathbb{R}_{+}^{T^{2} \times Y^{m}}$ be nonempty, and $\mathcal{S}: E \rightarrow \mathbb{R}_{+}^{T \times Y^{m}}$. The conditional functional equation for mappings $f \in Y^{T}$

$$
f(s+t)=f(s)+f(t), \quad \text { for every } s, t \in T \text { such that } s+t \in T,
$$

is said to be $\mathcal{S}$-stable if for any $\psi \in Y^{T}$ and any $\delta \in E$ with

$$
\|\psi(s+t)-\psi(t)-\psi(s), y\| \leq \delta(s, t, y), \quad \text { for every } y \in Y^{m} \text { and } s, t \in T \text { such that } s+t \in T,
$$

there exists a solution $\phi \in Y^{T}$ of Equation (5) such that

$$
\|\phi(t)-\psi(t), y\| \leq(\mathcal{S} \delta)(t, y), \quad \text { for every } t \in T \text { and } y \in Y^{m}
$$

Let us mention that functional Equation (5) is conditional, because it can be rewritten in the following conditional form:

$$
\text { if } s+t \in T \text {, then } f(s+t)=f(s)+f(t) \text {. }
$$

In the very particular case, when $(\mathcal{S} \delta)(t, y)=0$ for every $\delta \in E, t \in T$ and $y \in Y^{m}$, the $\mathcal{S}$-stability is called hyperstability (see [30] for more details). Specifically, we have the following definition.

Definition 2. Let $(P,+)$ be a groupoid, $T \neq \varnothing$ be a subset of $P$ and $E \subset \mathbb{R}^{T^{2} \times Y^{m}}$ be nonempty. The conditional Equation (5) is said to be E-hyperstable if every $\psi \in Y^{T}$ satisfying (6) with some $\delta \in E$, is a solution of Equation (5).

In this paper, we investigate the hyperstabilty case for Equation (5). An example of such hyperstability results for classical normed spaces, motivated by some earlier well-known outcomes of Th.M. Rassias [29,31] (see also [9,32]) and J.M. Rassias [11,12], is the following main theorem in [13] (we write $\mathbb{N}_{k_{0}}:=\left\{n \in \mathbb{N}: n \geq k_{0}\right\}$ for $k_{0} \in \mathbb{N}$ ).

Theorem 2. Let $E_{1}$ and $E_{2}$ be classic normed spaces, $\varnothing \neq X \subset E_{1} \backslash\{0\}, c \geq 0$, and $p, q$ be real numbers with $p+q<0$. Suppose that there exists $k_{0} \in \mathbb{N}$ such that

$$
k z \in X, \quad \text { for every } k \in \mathbb{N}_{k_{0}} \text { and } z \in X \text {. }
$$

Then each mapping $f: X \rightarrow E_{2}$ with

$$
\left\|f\left(x_{1}+x_{2}\right)-f\left(x_{1}\right)-f\left(x_{2}\right)\right\| \leq c\left\|x_{1}\right\|^{q}\left\|x_{2}\right\|^{p}, \quad \text { for every } x_{1}, x_{2} \in X \text { such that } x_{1}+x_{2} \in X,
$$

is additive on $X$.

The next two theorems present two possible ways to extend Theorem 2 to the case of $n$-normed spaces. They are the main results of this paper. Their proofs are provided in the next section.

Theorem 3. Let $\left(H,\|\cdot\|_{*}\right)$ be a normed space, $X \subset H \backslash\{0\}$ be nonempty, $Y_{0}:=Y \backslash\{0\}, \mu: Y_{0}^{m} \rightarrow \mathbb{R}_{+}$, and $p, q \in \mathbb{R}, p+q<0$. Assume that there exists $k_{0} \in \mathbb{N}$ such that (7) holds. Then every mapping $f: X \rightarrow Y$ satisfying the inequality

$$
\begin{array}{r}
\left\|f\left(x_{1}+x_{2}\right)-f\left(x_{1}\right)-f\left(x_{2}\right), y\right\| \leq\left\|x_{1}\right\|_{*}^{q}\left\|x_{2}\right\|_{*}^{p} \mu(y), \quad \text { for every } y \in Y_{0}^{m} \text { and } x_{1}, x_{2} \in X \\
\text { such that } x_{1}+x_{2} \in X,
\end{array}
$$

is additive on $X$. 
Theorem 4. Let $\operatorname{dim} Y \geq m+3, X \subset Y$ be nonempty, $c, p, q \in \mathbb{R}, p+q \neq 1$ and $c \geq 0$. Then every mapping $g: X \rightarrow Y$ satisfying the inequality

$$
\left\|g\left(x_{1}+x_{2}\right)-g\left(x_{1}\right)-g\left(x_{2}\right), y\right\| \leq c\left\|x_{1}, y\right\|^{q}\left\|x_{2}, y\right\|^{p}
$$

for every $y \in Y^{m}$ and every $x_{1}, x_{2} \in X$ with $x_{1}+x_{2} \in X$ and $\left\|x_{1}, y\right\|\left\|x_{2}, y\right\| \neq 0$, is additive on $X$.

Remark 2. If $\mathfrak{a}=\left(a_{1}, \ldots, a_{m+1}\right)$ is a sequence of linearly independent vectors in $Y$, then it is easy to check that the formula

$$
\|x\|_{\mathfrak{a}}:=\sum_{j=1}^{m+1}\left\|b_{1 j}, \ldots, b_{m+1 j}\right\|, \quad \text { for every } x \in Y,
$$

defines a norm in $Y$, where $b_{i j}=a_{i}$ if $i \neq j$ and $b_{j j}=x$ (cf. Remark 1$)$. Thus, we see that the $(m+1)$-norm in $Y$ generates a large family of norms in $Y$.

Let $\|\cdot\|_{1}, \ldots,\|\cdot\|_{m}$ be norms in $Y$; they can be chosen from the norms generated by the $(m+1)$-norm in the way described above. Let $r_{1}, \ldots, r_{m} \in \mathbb{R}, c, c_{1}, \ldots, c_{m} \in \mathbb{R}_{+}$and $Y_{0}:=Y \backslash\{0\}$. Then we can use in (9) the following two natural examples of function $\mu: Y_{0}^{m} \rightarrow \mathbb{R}$ :

$$
\begin{aligned}
& \mu(y)=c \prod_{i=1}^{m}\left\|y_{i}\right\|_{i}^{r_{i}}, \quad \text { for every } y=\left(y_{1}, \ldots, y_{m}\right) \in Y_{0}^{m}, \\
& \mu(y)=\sum_{i=1}^{m} c_{i}\left\|y_{i}\right\|_{i}^{r_{i}}, \quad \text { for every } y=\left(y_{1}, \ldots, y_{m}\right) \in Y_{0}^{m} .
\end{aligned}
$$

We end this section with two examples of simple corollaries that can be derived from Theorem 4.

Corollary 1. Assume that $Y$ and $c, p, q$ are as in Theorem 4 and $F: Y^{2} \rightarrow Y$ satisfies the inequality

$$
\left\|F\left(x_{1}, x_{2}\right), y\right\| \leq c\left\|x_{1}, y\right\|^{q}\left\|x_{2}, y\right\|^{p}
$$

for every $y \in Y^{m}$ and every $x_{1}, x_{2} \in Y$ such that $\left\|x_{1}, y\right\|\left\|x_{2}, y\right\| \neq 0$. Then the functional equation

$$
g\left(x_{1}+x_{2}\right)=g\left(x_{1}\right)+g\left(x_{2}\right)+F\left(x_{1}, x_{2}\right)
$$

has at least one solution $g: Y \rightarrow Y$ if and only if

$$
F\left(x_{1}, x_{2}\right)=0, \quad \text { for every } x_{1}, x_{2} \in Y .
$$

Proof. Suppose that there exists a solution $g: Y \rightarrow Y$ of Equation (12). Then

$$
g\left(x_{1}+x_{2}\right)-g\left(x_{1}\right)-g\left(x_{2}\right)=F\left(x_{1}, x_{2}\right), \quad \text { for every } x_{1}, x_{2} \in Y,
$$

and consequently

$$
\left\|g\left(x_{1}+x_{2}\right)-g\left(x_{1}\right)-g\left(x_{2}\right), y\right\|=\left\|F\left(x_{1}, x_{2}\right), y\right\| \leq c\left\|x_{1}, y\right\|^{q}\left\|x_{2}, y\right\|^{p}
$$

for every $y \in Y^{m}$ and every $x_{1}, x_{2} \in Y$ such that $\left\|x_{1}, y\right\|\left\|x_{2}, y\right\| \neq 0$. Consequently, by Theorem $4, g$ is additive, which means that $F\left(x_{1}, x_{2}\right)=g\left(x_{1}+x_{2}\right)-g\left(x_{1}\right)-g\left(x_{2}\right)=0$ for every $x_{1}, x_{2} \in Y$.

The converse is trivial. Specifically, if $F\left(x_{1}, x_{2}\right)=0$ for every $x_{1}, x_{2} \in Y$, then the function $g: Y \rightarrow Y, g(x)=0$ for $x \in Y$, is a solution of Equation (12). 
Corollary 2. Assume that $Y$ and $c, p, q$ are as in Theorem 4. Let $F: Y^{2} \rightarrow Y$ be a solution to the cocycle functional equation

$$
\begin{gathered}
F\left(x_{1}, x_{2}\right)+F\left(x_{1}+x_{2}, x_{3}\right)=F\left(x_{1}, x_{2}+x_{3}\right)+F\left(x_{2}, x_{3}\right), \quad \text { for every } x_{1}, x_{2}, x_{3} \in Y, \\
F\left(x_{1}, x_{2}\right)=F\left(x_{2}, x_{1}\right), \quad \text { for every } x_{1}, x_{2} \in Y
\end{gathered}
$$

and inequality (11) be valid for every $y \in Y^{m}$ and every $x_{1}, x_{2} \in Y$ such that $\left\|x_{1}, y\right\|\left\|x_{2}, y\right\| \neq 0$. Then (13) holds.

Proof. According to [33] (Theorem 1), a mapping $F: Y^{2} \rightarrow Y$ is a solution to Equations (14) and (15) if and only if there exists a mapping $g: Y \rightarrow Y$ with

$$
F\left(x_{1}, x_{2}\right)=g\left(x_{1}+x_{2}\right)-g\left(x_{1}\right)-g\left(x_{2}\right), \quad \text { for every } x_{1}, x_{2} \in Y .
$$

The rest of the proof is analogous as for Corollary 4.

Similar results can be deduced from Theorem 3 .

\section{Proofs of Theorems 3 and 4}

For the proof of Theorem 3 we need an auxiliary fixed-point theorem that can be easily derived from the main result in [34]. To present it we introduce the following two hypotheses:

(A1) $E$ is a nonempty set, $j \in \mathbb{N}, L_{1}, \ldots, L_{j}: E \times Y^{m} \rightarrow \mathbb{R}_{+}$and $f_{1}, \ldots, f_{j}: E \rightarrow E$.

(A2) $\Lambda: \mathbb{R}_{+}^{E \times Y^{m}} \rightarrow \mathbb{R}_{+}^{E \times Y^{m}}$ is an operator defined by

$$
\Lambda \delta(x, y):=\sum_{i=1}^{j} L_{i}(x, y) \delta\left(f_{i}(x), y\right), \quad \text { for every } \delta \in \mathbb{R}_{+}^{E \times Y^{m}}, x \in E \text { and } y \in Y^{m} .
$$

We say that $\mathcal{T}: Y^{E} \rightarrow Y^{E}$ is $\Lambda-$ contractive if

$$
\|\mathcal{T} \xi(x)-\mathcal{T} \mu(x), y\| \leq \Lambda \delta(x, y), \quad \text { for every } x \in E \text { and } y \in Y^{m},
$$

for any $\xi, \mu \in Y^{E}$ and $\delta \in \mathbb{R}_{+}^{E \times Y^{m}}$ with

$$
\|\xi(x)-\mu(x), y\| \leq \delta(x, y), \quad \text { for every } x \in E \text { and } y \in Y^{m} .
$$

For given set $A \neq \varnothing$ and $h \in A^{A}$, we define $h^{n} \in A^{A}$ for $n \in \mathbb{N}_{0}:=\mathbb{N} \cup\{0\}$ by:

$$
h^{0}(x)=x, \quad h^{n+1}(x)=h\left(h^{n}(x)\right), \quad \text { for every } x \in A \text { and } n \in \mathbb{N}_{0} .
$$

The fixed-point theorem reads as follows.

Theorem 5. Let (A1) and (A2) be valid and $\mathcal{T}: Y^{E} \rightarrow Y^{E}$ be $\Lambda$-contractive. Let mappings $\varepsilon \in \mathbb{R}^{E \times Y^{m}}$ and $\varphi \in Y^{E}$ be such that

$$
\begin{aligned}
& \|\mathcal{T} \varphi(x)-\varphi(x), y\| \leq \varepsilon(x, y), \quad \text { for every } x \in E \text { and } y \in Y^{m} \\
& \varepsilon^{*}(x, y):=\sum_{i=0}^{\infty} \Lambda^{i} \varepsilon(x, y)<\infty, \quad \text { for every } x \in E \text { and } y \in Y^{m} .
\end{aligned}
$$


Then, for each $x \in E$, there exists the limit

$$
\psi:=\lim _{n \rightarrow \infty} \mathcal{T}^{n} \varphi(x)
$$

and the function $\psi \in Y^{E}$, defined in this way, is a unique fixed point of $\mathcal{T}$ with

$$
\|\varphi(x)-\psi(x), y\| \leq \varepsilon^{*}(x, y), \quad \text { for every } x \in E \text { and } y \in Y^{m} .
$$

Now, we are able to prove Theorem 3.

Proof of Theorem 3. Let

$$
W(x, z, y):=\|x\|_{*}^{q}\|z\|_{*}^{p} \mu(y)
$$

for $y \in Y_{0}^{m}$ and $x, z \in X$. From the above it follows that:

$$
\begin{aligned}
& W(n x, z, y)=n^{q} W(x, z, y), \quad W(x, n z, y)=n^{p} W(x, z, y), \\
& \quad \text { for every } x, z \in X, n \in \mathbb{N}_{k_{0}} \text { and } y \in Y_{0}^{m} .
\end{aligned}
$$

Please note that we must have $p<0$ or $q<0$, because $p+q<0$. We consider only the case where $p<0$; the case $q<0$ is analogous.

Let $f: X \rightarrow Y$ be a mapping satisfying (9). Fix $x_{1}, x_{2} \in X$ with $x_{1}+x_{2} \in X$ and take $l \in \mathbb{N}_{k_{0}}$ such that

$$
l^{p+q}+(1+l)^{p+q}<1 .
$$

It is easy to see that (9) with $x_{2}=l x_{1}$ gives

$$
\left\|f\left((l+1) x_{1}\right)-f\left(x_{1}\right)-f\left(l x_{1}\right), y\right\| \leq W\left(x_{1}, l x_{1}, y\right), \quad \text { for every } x_{1} \in X \text { and } y \in Y^{m} .
$$

Define operators $\mathcal{T}: Y^{X} \rightarrow Y^{X}$ and $\Lambda: \mathbb{R}_{+}^{X} \rightarrow \mathbb{R}_{+}^{X}$ by

$$
\begin{gathered}
\mathcal{T} \xi(x):=\xi((l+1) x)-\xi(l x), \quad \text { for every } x \in X \text { and } \xi \in Y^{X}, \\
\Lambda \delta(x, y):=\delta((l+1) x, y)+\delta(l x, y), \quad \text { for every } x \in X, y \in Y^{m} \text { and } \delta \in \mathbb{R}_{+}^{X \times Y^{m}} .
\end{gathered}
$$

Then $\Lambda$ has the form as in (A2) with $j=2, f_{1}(x)=(l+1) x, f_{2}(x)=l x, L_{1}(x, y)=L_{2}(x, y)=1$ for $x \in X$ and $y \in Y^{m}$ and $\mathcal{T}$ is $\Lambda$-contractive. Please note that (18) takes the form

$$
\|\mathcal{T} f(x)-f(x), y\| \leq l^{p} W(x, x, y), \quad \text { for every } x \in X \text { and } y \in Y^{m} .
$$

Let $\varepsilon(x, y):=l^{p} W(x, x, y)$ for $x \in X$ and $y \in Y^{m}$. Then, by (17) (with $x=z$ ), we have

$$
\varepsilon(k x, y)=k^{p+q} \varepsilon(x, y), \quad \text { for every } x \in X, y \in Y^{m} \text { and } k \in \mathbb{N}_{k_{0}} .
$$

Next, from (20) and (21), by an easy induction, it follows that for each $n \in \mathbb{N}_{0}$,

$$
\Lambda^{n} \varepsilon(x, y) \leq\left((1+l)^{p+q}+l^{p+q}\right)^{n} \varepsilon(x, y), \quad \text { for every } x \in X \text { and } y \in Y^{m} .
$$

Hence, from the geometric queue summation, it results that

$$
\begin{aligned}
\varepsilon^{*}(x, y) & :=\sum_{n=0}^{\infty} \Lambda^{n} \varepsilon(x, y) \leq \varepsilon(x, y) \sum_{n=0}^{\infty}\left((1+l)^{p+q}+l^{p+q}\right)^{n} \\
& =\frac{\varepsilon(x, y)}{1-l^{p+q}-(1+l)^{p+q}}, \quad \text { for every } x \in X \text { and } y \in Y^{m} .
\end{aligned}
$$

Thus, we see that (16) is valid. 
Consequently, by Theorem 5 , there is a solution $T_{l}: X \rightarrow Y$ of the equation

$$
T(x)=T((1+l) x)-T(l x)
$$

such that

$$
\left\|f(x)-T_{l}(x), y\right\| \leq \frac{l^{p} W(x, x, y)}{1-l^{p+q}-(1+l)^{p+q}}, \quad \text { for every } x \in X \text { and } y \in Y^{m} .
$$

Moreover,

$$
T_{l}(x):=\lim _{n \rightarrow \infty} \mathcal{T}^{n} f(x), \quad \text { for every } x \in X .
$$

Now we show by induction that for every $x_{1}, x_{2} \in X$ with $x_{1}+x_{2} \in X$ and $n \in \mathbb{N}_{0}$,

$$
\begin{aligned}
\| \mathcal{T}^{n} f\left(x_{1}\right. & \left.+x_{2}\right)-\mathcal{T}^{n} f\left(x_{1}\right)-\mathcal{T}^{n} f\left(x_{2}\right), y \| \\
& \leq\left((l+1)^{p+q}+l^{p+q}\right)^{n} W\left(x_{1}, x_{2}, y\right), \quad \text { for every } y \in Y^{m}
\end{aligned}
$$

The case $n=0$ is just (9). Next, fix $k \in \mathbb{N}_{0}$ and assume that (24) holds for $n=k$ and for every $x_{1}, x_{2} \in X$ with $x_{1}+x_{2} \in X$. Then, by (22) and the triangle inequality, for every $x_{1}, x_{2} \in X$ with $x_{1}+x_{2} \in X$ we have

$$
\begin{aligned}
& \left\|\mathcal{T}^{k+1} f\left(x_{1}+x_{2}\right)-\mathcal{T}^{k+1} f\left(x_{1}\right)-\mathcal{T}^{k+1} f\left(x_{2}\right), y\right\| \\
& \leq\left\|\mathcal{T}^{k} f\left((l+1) x_{1}+(l+1) x_{2}\right)-\mathcal{T}^{k} f\left((l+1) x_{1}\right)-\mathcal{T}^{k} f\left((l+1) x_{2}\right), y\right\| \\
& \quad+\left\|\mathcal{T}^{k} f\left(l x_{1}+l x_{2}\right)-\mathcal{T}^{k} f\left(l x_{1}\right)-\mathcal{T}^{k} f\left(l x_{2}\right), y\right\|, \quad \text { for every } y \in Y^{m}
\end{aligned}
$$

whence and by (17) we finally get

$$
\begin{aligned}
\| \mathcal{T}^{k+1} f\left(x_{1}+\right. & \left.x_{2}\right)-\mathcal{T}^{k+1} f\left(x_{1}\right)-\mathcal{T}^{k+1} f\left(x_{2}\right), y \| \\
\leq & \left(l^{p+q}+(1+l)^{p+q}\right)^{k} W\left((l+1) x_{1},(l+1) x_{2}, y\right) \\
& +\left(l^{p+q}+(1+l)^{p+q}\right)^{k} W\left(l x_{1}, l x_{2}, y\right) \\
= & \left(l^{p+q}+(1+l)^{p+q}\right)^{k+1} W\left(x_{1}, x_{2}, y\right), \quad \text { for every } y \in Y^{m}
\end{aligned}
$$

This completes the proof of (24).

Letting $n \rightarrow \infty$ in (24), we obtain

$$
\left\|T_{l}\left(x_{1}+x_{2}\right)-T_{l}\left(x_{1}\right)-T_{l}\left(x_{2}\right), y\right\|=0
$$

for every $y \in Y^{m}$ and every $x_{1}, x_{2} \in X$ with $x_{1}+x_{2} \in X$. Thus, we have proved that (see Remark 1 )

$$
T_{l}\left(x_{1}+x_{2}\right)=T_{l}\left(x_{1}\right)+T_{l}\left(x_{2}\right), \quad \text { for every } x_{1}, x_{2} \in X \text { with } x_{1}+x_{2} \in X .
$$

Next we show that $T_{l}$ is the unique function mapping $X$ into $Y$ satisfying (26) and such that there is $S>0$ with

$$
\left\|f(x)-T_{l}(x), y\right\| \leq S \varepsilon(x, y), \quad \text { for every } x \in X \text { and } y \in Y^{m} .
$$

Suppose that $T_{0}: X \rightarrow Y$ satisfies

$$
T_{0}\left(x_{1}+x_{2}\right)=T_{0}\left(x_{1}\right)+T_{0}\left(x_{2}\right), \quad \text { for every } x_{1}, x_{2} \in X \text { with } x_{1}+x_{2} \in X,
$$

and there exists $B_{0} \in(0, \infty)$ with

$$
\left\|f(x)-T_{0}(x), y\right\| \leq B_{0} \varepsilon(x, y), \quad \text { for every } x \in X \text { and } y \in Y^{m} .
$$


Then

$$
\begin{aligned}
\left\|T_{l}(x)-T_{0}(x), y\right\| & \leq\left\|T_{l}(x)-f(x), y\right\|+\left\|f(x)-T_{0}(x), y\right\| \leq\left(B_{0}+S\right) \varepsilon(x, y) \\
& =B \varepsilon(x, y) \sum_{n=0}^{\infty}\left(l^{p+q}+(1+l)^{p+q}\right)^{n}, \quad \text { for every } x \in X \text { and } y \in Y^{m},
\end{aligned}
$$

where according to the summation of geometric queue $B:=\left(B_{0}+S\right)\left(1-l^{p+q}-(1+l)^{p+q}\right)$.

Next we prove that for each $\iota \in \mathbb{N}_{0}$

$$
\left\|T_{l}(x)-T_{0}(x), y\right\| \leq B \varepsilon(x, y) \sum_{n=l}^{\infty}\left(l^{p+q}+(1+l)^{p+q}\right)^{n}, \quad \text { for every } x \in X \text { and } y \in Y^{m} .
$$

Clearly, the case $\iota=0$ is exactly (28). Therefore, fix $k \in \mathbb{N}_{0}$ and assume that (29) holds for $\iota=k$. Then by (21), (26), (27) and the triangle inequality, we get

$$
\begin{aligned}
\| T_{l}(x) & -T_{0}(x), y\|=\| T_{l}((1+l) x)-T_{l}(l x)-T_{0}((1+l) x)+T_{0}(l x), y \| \\
& \leq\left\|T_{l}((1+l) x)-T_{0}((1+l) x), y\right\|+\left\|T_{l}(l x)-T_{0}(l x), y\right\| \\
& \leq B(\varepsilon((1+l) x, y)+\varepsilon(l x, y)) \sum_{n=k}^{\infty}\left(l^{p+q}+(1+l)^{p+q}\right)^{n} \\
& \leq B\left((1+l)^{p+q} \varepsilon(x, y)+l^{p+q} \varepsilon(x, y)\right) \sum_{n=k}^{\infty}\left(l^{p+q}+(1+l)^{p+q}\right)^{n} \\
& =B \varepsilon(x, y) \sum_{n=k+1}^{\infty}\left(l^{p+q}+(1+l)^{p+q}\right)^{n}, \quad \text { for every } x \in X \text { and } y \in Y^{m} .
\end{aligned}
$$

This completes the inductive proof of (29). Now, letting $\iota \rightarrow \infty$ in (29), we get $T_{l}=T_{0}$. In the same way, for each $j \in \mathbb{N}$ we obtain a unique mapping $T_{l+j}: X \rightarrow Y$ such that

$$
T_{l+j}\left(x_{1}+x_{2}\right)=T_{l+j}\left(x_{1}\right)+T_{l+j}\left(x_{2}\right), \quad \text { for every } x_{1}, x_{2} \in X \text { with } x_{1}+x_{2} \in X,
$$

and

$$
\left\|f(x)-T_{l+j}(x), y\right\| \leq \frac{(l+j)^{p} W(x, x, y)}{1-(l+j)^{p+q}-(1+l+j)^{p+q}}, \quad \text { for every } x \in X \text { and } y \in Y^{m} .
$$

Furthermore, the uniqueness of $T_{l}$ implies that $T_{l}=T_{l+j}$ for each $j \in \mathbb{N}$. Hence

$$
\left\|f(x)-T_{l}(x), y\right\| \leq \frac{(l+j)^{p} W(x, x, y)}{1-(l+j)^{p+q}-(1+l+j)^{p+q}} .
$$

for every $x \in X$, every $y \in Y^{m}$ and every $j \in \mathbb{N}$. Consequently, since $p<0$ and $p+q<0$, letting $j \rightarrow \infty$ in (31), we obtain

$$
\left\|f(x)-T_{l}(x), y\right\|=0, \quad \text { for every } x \in X \text { and } y \in Y^{m},
$$

which means that $f=T_{l}$ and consequently (2) holds. This completes the proof. 
Remark 3. Please note that with only very small and obvious modification in the proof, we can replace condition (9) in Theorem 3 with the following one:

$$
\left\|f\left(x_{1}+x_{2}\right)-f\left(x_{1}\right)-f\left(x_{2}\right), y\right\| \leq W\left(x_{1}, x_{2}, y\right)
$$

where $W$ is any function mapping $X^{2} \times Y_{0}^{m}$ into $\mathbb{R}$ that fulfils the inequalities

$$
W(n x, z, y) \leq n^{q} W(x, z, y), \quad W(x, n z, y) \leq n^{p} W(x, z, y)
$$

for every $x, z \in X$, every $n \in \mathbb{N}_{k_{0}}$ and every $y \in Y_{0}^{m}$.

Finally, we prove Theorem 4.

Proof of Theorem 4. Let $g: X \rightarrow Y$ be a mapping satisfying inequality (10) for every $y \in Y^{m}$ and every $x_{1}, x_{2} \in X$ with $x_{1}+x_{2} \in X$ and $\left\|x_{1}, y\right\|\left\|x_{2}, y\right\| \neq 0$. Take $x_{1}, x_{2} \in X$ with $x_{1}+x_{2} \in X$. Since $\operatorname{dim} Y \geq m+3$, there exist $u_{1}, \ldots, u_{m+1}$ such that the sets of vectors $\left\{x_{1}, u_{1}, \ldots, u_{m+1}\right\}$ and $\left\{x_{2}, u_{1}, \ldots, u_{m+1}\right\}$ are linearly independent.

Fix $y_{1}, \ldots, y_{m} \in\left\{u_{1}, \ldots, u_{m+1}\right\}$ such that $y_{i} \neq y_{j}$ for $i \neq j$, where $i, j=1, \ldots, m$, and write $y:=\left(y_{1}, \ldots, y_{m}\right)$. Then, for each $a \in(0, \infty)$, the sets of vectors $\left\{x_{1}, a y_{1}, \ldots, a y_{m}\right\}$ and $\left\{x_{2}, a y_{1}, \ldots, a y_{m}\right\}$ are linearly independent, which means that $\left\|x_{1}, a y\right\|\left\|x_{2}, a y\right\| \neq 0$ (see (C1)). Consequently, by (10),

$$
\begin{aligned}
a^{m}\left\|g\left(x_{1}+x_{2}\right)-g\left(x_{1}\right)-g\left(x_{2}\right), y\right\| & =\left\|g\left(x_{1}+x_{2}\right)-g\left(x_{1}\right)-g\left(x_{2}\right), a y\right\| \\
& \leq c\left\|x_{1}, a y\right\|^{q}\left\|x_{2}, a y\right\|^{p} \\
& =a^{m(p+q)} c\left\|x_{1}, y\right\|^{q}\left\|x_{2}, y\right\|^{p}, \quad a \in(0, \infty) .
\end{aligned}
$$

This means that

$$
\left\|g\left(x_{1}+x_{2}\right)-g\left(x_{1}\right)-g\left(x_{2}\right), y\right\| \leq a^{m(p+q-1)} c\left\|x_{1}, y\right\|^{q}\left\|x_{2}, y\right\|^{p}, \quad a \in(0, \infty),
$$

whence $\left\|g\left(x_{1}+x_{2}\right)-g\left(x_{1}\right)-g\left(x_{2}\right), y\right\|=0$.

Thus, we have proved that $\left\|g\left(x_{1}+x_{2}\right)-g\left(x_{1}\right)-g\left(x_{2}\right), y_{1}, \ldots, y_{m}\right\|=0$ for every $y_{1}, \ldots, y_{m} \in$ $\left\{u_{1}, \ldots, u_{m+1}\right\}$ (the case where $y_{i}=y_{j}$ for some $i \neq j$ is trivial, because then vectors $y_{1}, \ldots, y_{m}$ are linearly dependent). Therefore, by Remark $1, g\left(x_{1}+x_{2}\right)-g\left(x_{1}\right)-g\left(x_{2}\right)=0$.

\section{Conclusions}

In this article, we presented two possible hyperstability results for the Cauchy functional equation $f(x+y)=f(x)+f(y)$ on a restricted domain, for mappings that take values in an $n$-normed space. They correspond to some earlier classical results obtained for normed spaces. Moreover, the second one (Theorem 4), even if quite simple, could be described as somewhat unexpected, because the assumptions that $Y$ is complete and $p+q<0$ are not necessary there.

Author Contributions: Conceptualization, J.B. and E.-s.E.-h.; methodology, J.B. and E.-s.E.-h.; software, J.B. and E.-s.E.-h.; validation, J.B. and E.-s.E.-h.; formal analysis, J.B. and E.-s.E.-h.; investigation, J.B. and E.-s.E.-h.; resources, J.B. and E.-s.E.-h.; data curation, J.B. and E.-s.E.-h.; writing-original draft preparation, E.-s.E.-h.; writing-review and editing, J.B. and E.-s.E.-h.; visualization, J.B. and E.-s.E.-h.; supervision, J.B.; project administration, E.-s.E.-h.; funding acquisition, J.B. and E.-s.E.-h. All authors have read and agreed to the published version of the manuscript.

Funding: This research received no external funding.

Conflicts of Interest: The authors declare no conflict of interest. 


\section{References}

1. Hyers, D.H. On the stability of the linear functional equation. Proc. Nat. Acad. Sci. USA 1941, $27,222-224$. [CrossRef]

2. Hyers, D.H.; Isac, G.; Rassias, T.M. Stability of Functional Equations in Several Variables; Birkhäuser: Boston, MA, USA, 1998.

3. Jung, S.-M.; Popa, D.; Rassias, M.T. On the stability of the linear functional equation in a single variable on complete metric groups. J. Global Optim. 2014, 59, 165-171. [CrossRef]

4. Moszner, Z. Stability has many names. Aequ. Math. 2016, 90, 983-999. [CrossRef]

5. Brzdęk, J.; Popa, D.; Raşa, I. Hyers-Ulam stability with respect to gauges. J. Math. Anal. Appl. 2017, 453, 620-628. [CrossRef]

6. Elqorachi, E.; Rassias, M.T. Generalized Hyers-Ulam stability of trigonometric functional equations. Mathematics 2018, 6, 83. [CrossRef]

7. Brzdęk, J.; Popa, D.; Raşa, I.; Xu, B. Ulam Stability of Operators; Academic Press, Elsevier: Oxford, MA, USA, 2018.

8. Brzdęk, J.; Fechner, W.; Moslehian, M.S.; Sikorska, J. Recent developments of the conditional stability of the homomorphism equation. Banach J. Math. Anal. 2015, 9, 278-326. [CrossRef]

9. Rassias, T.M.; Šemrl, P. On the behavior of mappings which do not satisfy Hyers-Ulam stability. Proc. Amer. Math. Soc. 1992, 114, 989-993. [CrossRef]

10. Brzdęk, J. Note on stability of the Cauchy equation-An answer to a problem of Th.M. Rassias. Carpat. J. Math. 2014, 30, 47-54.

11. Rassias, J.M. On approximation of approximately linear mappings by linear mappings. J. Funct. Anal. 1982, 46, 126-130. [CrossRef]

12. Rassias, J.M. On a new approximation of approximately linear mappings by linear mappings. Discuss. Math. 1985, 7, 193-196.

13. Brzdęk, J. A hyperstability result for the Cauchy equation. Bull. Aust. Math. Soc. 2014, 89, 33-40. [CrossRef]

14. Misiak, A. n-inner product spaces. Math. Nachr. 1989, 140, 299-319. [CrossRef]

15. Chu, H.-Y.; Kim, A.; Park, J. On the Hyers-Ulam stabilities of functional equations on n-Banach spaces. Math. Nachr. 2016, 289, 1177-1188. [CrossRef]

16. Gähler, S. Lineare 2-normierte Räume. Math. Nachr. 1964, 28, 1-43. [CrossRef]

17. Chung, S.C.; Park, W.G. Hyers-Ulam stability of functional equations in 2-Banach spaces. Int. J. Math. Anal. (Ruse) 2012, 6, 951-961.

18. Freese, R.W.; Cho, Y.J. Geometry of Linear 2-Normed Spaces; Nova Science Publishers, Inc.: New York, NY, USA, 2001.

19. Gao, J. On the stability of the linear mapping in 2-normed spaces. Nonlinear Funct. Anal. Appl. 2009, 14, 801-807.

20. Park, W.G. Approximate additive mappings in 2-Banach spaces and related topics. J. Math. Anal. 2011, 376, 193-202. [CrossRef]

21. Gunawan, H.; Mashadi, M. On n-normed spaces. Int. J. Math. Math. Sci. 2001, 27, 631-639. [CrossRef]

22. $\mathrm{Xu}, \mathrm{T} . Z$. Approximate multi-Jensen, multi-Euler-Lagrange additive and quadratic mappings in n-Banach spaces. Abstr. Appl. Anal. 2013, 2013, 648709. [CrossRef]

23. Xu, T.Z.; Rassias, J.M. On the Hyers-Ulam stability of a general mixed additive and cubic functional equation in n-Banach spaces. Abstr. Appl. Anal. 2012, 2012, 926390. [CrossRef]

24. Chen, X.Y.; Song, M.M. Characterizations on isometries in linear n-normed spaces. Nonlinear Anal. 2010, 72, 1895-1901. [CrossRef]

25. Chu, H.-Y.; Lee, K.; Park, C.-G. On the Aleksandrov problem in linear n-normed spaces. Nonlinear Anal. 2004, 59, 1001-1011.

26. Dutta, H. On some n-normed linear space valued difference sequences. J. Franklin Inst. 2011, 348, $2876-2883$. [CrossRef]

27. Eghbali, N.; Rassias, J.M.; Taheri, M. On the stability of a k-cubic functional equation in intuitionistic fuzzy n-normed spaces. Result. Math. 2016, 70, 233-248. [CrossRef]

28. Gunawan, H.; Neswan, O.; Sukaesih, E. Fixed point theorems in bounded sets in an n-normed space. J. Math. Anal. 2015, 6, 51-58.

29. Rassias, T.M. On a modified Hyers-Ulam sequence. J. Math. Anal. Appl. 1991, 158, 106-113. [CrossRef]

30. Brzdęk, J.; Ciepliński, K. Hyperstability and superstability. Abstr. Appl. Anal. 2013, 2013, 401756. [CrossRef] 
31. Rassias, T.M. On the stability of the linear mapping in Banach spaces. Proc. Amer. Math. Soc. 1978, 72, $297-300$. [CrossRef]

32. Aoki, T. On the stability of the linear transformation in Banach spaces. J. Math. Soc. Japan 1950, 2, 64-66. [CrossRef]

33. Jessen, B.; Karpf, J.; Thorup, A. Some functional equations in groups and rings. Math. Scand. 1968, 22, $257-265$. [CrossRef]

34. Brzdęk, J.; Ciepliński, K. A fixed point theorem in n-Banach spaces and Ulam stability. J. Math. Anal. Appl. 2019, 470, 632-646. [CrossRef]

Publisher's Note: MDPI stays neutral with regard to jurisdictional claims in published maps and institutional affiliations.

(C) 2020 by the authors. Licensee MDPI, Basel, Switzerland. This article is an open access article distributed under the terms and conditions of the Creative Commons Attribution (CC BY) license (http://creativecommons.org/licenses/by/4.0/). 